\title{
Evaluación de la resistencia a abolladura por cortante de vigas armadas híbridas de acero según la norma venezolana COVENIN 1618:1998
}

\section{Assessment of shear buckling resistance of hybrid girders according to the Venezuelan code COVENIN 1618:1998}

R. Chacón ${ }^{(*)}$, J. E. Rojas-Blonval ${ }^{(*)}$

\section{RESUMEN}

En este estudio se presenta un análisis de la resistencia a abolladura por cortante de vigas armadas híbridas según la norma venezolana COVENIN 1618:1998. El estudio se basa en una recopilación bibliográfica de ensayos experimentales encontrados así como en una base de datos numérica creada por los autores. Se comparan los valores obtenidos tanto experimental, como numéricamente con los derivados de la formulación teórica recogida en dicha normativa. Asimismo, dentro de la comparación, se incluyen los resultados derivados de la aplicación del Eurocódigo 3(EN1993-1-5) y las normativas americanas AISC-AASHTO. Los resultados que arrojan dichas comparaciones muestran el carácter conservador de COVENIN 1618:1998 en la valoración de dicha resistencia. En base a los resultados obtenidos, se proponen expresiones de diseño que permiten incluir el diseño híbrido de vigas armadas obteniendo del mismo su máximo beneficio estructural.

Palabras clave: Vigas híbridas; COVENIN 1618:1998; abolladura por cortante; Vigas armadas.

\section{ABSTRACT}

In this paper, an in-depth analysis of the structural response of hybrid steel plate girders subjected to shear forces is presented. The analysis is focused on the Venezuelan Code for Steel Structures COVENIN 1618:1998. The study is based upon a review of experimental tests as well as upon a numerical study carried out by the authors. Both experimental and numerical results are compared with the results provided by the aforementioned Code. Likewise, other relevant Codes such as EN1993-1-5 and AISC-AASHTO are employed for the sake of comparison. The results obtained show that the values given by COVENIN 1618:1998 concerning the shear resistance of hybrid steel plate girders are particularly conservative. A design proposal that may enhance the current COVENIN formulation in this particular topic is presented accordingly.

Keywords: Hybrid girders; COVENIN 1618:1998; shear buckling; Plate girders.

(*) Camins-Barcelona Tech, Universitat Politècnica de Catalunya, Barcelona (España).

Persona de contacto/Corresponding author: rolando.chacon@upc.edu (R. Chacón)

Cómo citar este artículo/Citation: Chacón, R., Rojas-Blonval, J. E. (2015). Evaluación de la resistencia a abolladura por cortante de vigas armadas híbridas de acero según la norma venezolana COVENIN 1618:1998. Informes de la Construcción, 67(538): eo75, doi: http://dx.doi.org/10.3989/ic.13.111.

Licencia / License: Salvo indicación contraria, todos los contenidos de la edición electrónica de Informes de la Construcción se distribuyen bajo una licencia de uso y distribución Creative Commons Reconocimiento no Comercial 3.o. España (cc-by-nc). 


\section{INTRODUCCIÓN}

Las vigas armadas están compuestas por chapas que conforman sus alas y almas, añadiéndose rigidizadores transversales y/o longitudinales allí donde se requiera. Las vigas armadas son necesarias en estructuras donde los perfiles laminados no ofrecen suficientes prestaciones y es necesario confeccionar elementos a medida que cumplan dichos requisitos estructurales. Si el acero de alas y almas es distinto, la misma se denomina viga armada híbrida. Las vigas híbridas se conciben para incrementar la resistencia a flexión sin incrementar sustancialmente el peso. Para ello, se dispone un acero de mayores prestaciones resistentes en las alas. Se puede decir que una viga armada homogénea con cierto espesor de ala puede transformarse en una viga armada híbrida aumentando el límite elástico del acero y disminuyendo el espesor de la chapa. Para poder lograr de manera eficiente dicha reducción, se deben verificar otros fenómenos de inestabilidad que se asocian al empleo de chapas esbeltas tales como abolladura por cortante, pandeo lateral o abolladura local. La utilización de vigas híbridas en la construcción de puentes metálicos resulta atractiva, ya que por lo general este tipo de estructuras se diseñan para salvar grandes luces y por tanto están sometidas a grandes momentos flectores.

En Venezuela se espera se lleven a cabo diversos proyectos de infraestructura relacionados con vialidad y obras de ferrocarril de importancia estratégica para el país. Se prevé que en la próxima década se construyan más de tres mil kilómetros entre carreteras y vías férreas (1).

Actualmente, la normativa vigente en Venezuela para el cálculo de estructuras en acero es COVENIN-MINDUR 16181998 (2). Se basa fundamentalmente en las normativas AISC (3) y AASHTO (4) las cuales son objeto de actualizaciones. Actualmente en COVENIN-MINDUR, se penaliza la utilización de vigas híbridas y se limita su resistencia a la abolladura a valores notablemente conservadores. Se ha observado en este punto una falta de actualización de la normativa ya que las normativas americanas sí se han actualizado.

El principal objetivo de esta investigación es presentar una propuesta de actualización del apartado 17.3 (a) de COVENINMINDUR 1618-1998. Para demostrar la factibilidad de dicha actualización, se realiza un estudio paramétrico con herramientas numéricas así como una recopilación bibliográfica exhaustiva. La propuesta puede traducirse en plantear de manera eficiente la utilización de vigas híbridas en la construcción de puentes u otras estructuras de gran envergadura, alternativa que ya ha sido desarrollada con creces en diferentes países (5).

\section{ESTADO DEL ARTE}

\subsection{Vigas armadas híbridas}

Las vigas armadas híbridas han sido objeto de estudio profuso a nivel mundial desde los años 40. Desde entonces se han realizado estudios relativos a sus ventajas e inconvenientes estructurales (5) (6), a su comportamiento a flexión (7) (8) (9), a su comportamiento a cargas concentradas (10), a fatiga (11), a solicitaciones de cortante (12) (13) (14) junto con otras investigaciones de carácter proyectual (15). Existen trabajos realizados por los autores que describen dichas investigaciones (16). Las conclusiones principales son:
- Las vigas híbridas representan una alternativa eficiente a las vigas homogéneas pues permiten incrementar la resistencia seccional a flexión sin que esto implique aumentar su peso. Se logra incrementando el límite elástico $f_{y}$ de las alas pero manteniendo el límite elástico del alma con aceros convencionales (controlando adecuadamente el pandeo lateral).

- Las vigas híbridas se comportan similarmente a las homogéneas ante solicitaciones de cortante (se desarrollan los mismos mecanismos resistentes).

- La capacidad resistente a patch loading está determinada básicamente por la rigidez y resistencia del alma y únicamente por la rigidez del ala y no su resistencia (10).

\subsection{Abolladura por cortante y resistencia post-crítica}

La resistencia a abolladura por cortante en vigas armadas ha sido objeto de estudio en las últimas décadas. Timoshenko (17) planteó ecuaciones que daban solución al problema de la inestabilidad de una chapa sometida a cortante [1]. Dicha expresión plantea la tensión crítica de abolladura obtenida a partir de un modelo bidimensional elástico.

$$
\tau_{\text {cri }}=k_{v} \frac{\pi^{2} \mathrm{E}}{12\left(1-v^{2}\right)}\left(\frac{\mathrm{t}_{\mathrm{w}}}{h_{w}}\right)^{2}
$$

El valor de $k_{v}$ varía según las condiciones de contorno de la placa en estudio y la relación de aspecto del panel. Tanto para paneles cuyos bordes se encuentran simplemente apoyados [2] como para paneles cuyos bordes están empotrados [3] se han encontrado aproximaciones del coeficiente de abolladura $k_{v}$.

$$
\begin{gathered}
k_{v}=5,34+\frac{4}{\rho^{2}} \text { donde } \rho=\frac{\mathrm{a}}{\mathrm{h}_{\mathrm{w}}} \\
k_{v}=8,98+\frac{5,96}{\rho^{2}} \text { donde } \rho=\frac{\mathrm{a}}{\mathrm{h}_{\mathrm{w}}}
\end{gathered}
$$

Desde entonces, se han propuesto teorías que explican mecánicamente la respuesta estructural de una pieza sometida a dichas solicitaciones una vez se ha alcanzado el umbral crítico. Inicialmente, Basler (18) propuso la contribución postcrítica del alma cargada y posteriormente se refinaron los estudios generando el modelo del campo diagonal de tracciones (19) (20) y método del campo girado de tensiones (21).

El modelo de Basler (18) se basa en la suma de la capacidad crítica más la capacidad post-crítica ante la abolladura por cortante de un panel. Cuando el cortante en un panel rigidizado transversalmente alcanza el valor de cortante crítico, el alma abolla debido a la tensión principal de compresión. Una vez abolla se forma una banda diagonal de tracciones a lo largo del alma que se ancla en las alas y en los rigidizadores transversales en las esquinas opuestas del panel. La acción del campo de tracciones puede visualizarse como el mecanismo de una celosía tipo Pratt

Para el modelo de Cardiff se realizó una extensa campaña experimental la cual incluía paneles con distintas relaciones de aspecto así como un amplio rango de variación de la rigidez de las alas, con el fin de analizar su contribución (19) (20). Se propuso un mecanismo de colapso que conjuntamente con la contribución del alma (tension field), se basa en la formación de cuatro rotulas plásticas en las alas. 
Asimismo, Höglung (21) desarrolló el modelo del campo girado de tensiones. Este modelo tiene en cuenta que el alma luego de la abolladura presenta una resistencia post-crítica significativa, mediante esfuerzos de tracción anclados en las alas y rigidizadores transversales que la rodean. En un estado de cortante puro, el valor absoluto de las tensiones principales $\sigma_{1}$ y $\sigma_{2}$ es idéntico. Cuando se alcanza la tensión crítica de abolladura $\tau_{c r i}$, se produce una redistribución de tensiones.

Los modelos presentados constituyen la base teórica de las normativas actuales en Europa y los Estados Unidos. Existen publicaciones de carácter comparativo de los distintos modelos de resistencia a la abolladura en donde se valora profusamente la idoneidad de los mismos (22).

\section{NORMATIVAS}

A continuación se describen de manera sucinta las diferentes normativas utilizadas en la comparación realizada. Todas definen la resistencia a abolladura por cortante a partir de la configuración geométrica de la pieza y del material que la conforma. La Figura 1 muestra las variables geométricas que juegan un papel fundamental en dicho cálculo. Cabe destacar que las normativas tienen distinta nomenclatura. Con el fin de uniformizar las expresiones y cálculos, se plantea una nomenclatura única según lo que se establece en la Figura 1.

\subsection{COVENIN - MINDUR 1618-98}

La resistencia a abolladura por cortante de paneles de vigas armadas se define como $V_{t}$ y se calculará para el caso en el cual no se considera la resistencia post-crítica [4] y en el caso en el que sí se considera [5]. Se basa en la formulación propuesta por Basler.

$$
\begin{gathered}
V_{t}=0,6 t_{w} h_{w y w} f_{v} C_{v} \\
V_{t}=0,6 t_{w} h_{w} f_{y w}\left[C_{v}+\frac{1-C_{v}}{1,15 \sqrt{1+\left(\frac{a}{h w}\right)^{2}}}\right]
\end{gathered}
$$

$C_{v}$ representa la relación entre la tensión crítica de abolladura del alma y la tensión de plastificación por cortante del material del alma (Tabla 1$)$.

No se permitirá usar la acción del campo de tracción en los paneles extremos de las vigas armadas no híbridas, en todos los paneles de las vigas híbridas y en las vigas armadas de altura variable linealmente, y cuando la relación de aspecto del panel a/h $h_{w}$ exceda el menor valor entre 3,0 o $\left[260 /\left(h_{w} / t_{w}\right)\right]^{2}$.

\begin{tabular}{|c|c|c|}
\hline Condición & $\mathbf{C}_{\mathrm{v}}$ & $\mathbf{k}_{\mathrm{v}}$ \\
\hline$\frac{\mathrm{h}_{\mathrm{w}}}{\mathrm{t}_{\mathrm{w}}} \leq \alpha \sqrt{\frac{\mathrm{k}_{\mathrm{v}} \mathrm{E}}{\mathrm{f}_{\mathrm{yw}}}}$ & 1,0 & \multirow{3}{*}{$\mathrm{k}_{\mathrm{v}}-5+\frac{5}{\left(\frac{\mathrm{a}}{\mathrm{h}_{\mathrm{w}}}\right)^{2}}$} \\
\hline$\chi \cdot \sqrt{\frac{\mathrm{E}_{\mathrm{kv}}}{\mathrm{f}_{\mathrm{yw}}}}<\frac{\mathrm{h}_{\mathrm{w}}}{\mathrm{t}_{\mathrm{yw}}} \leq \sqrt{\frac{1}{\underline{\underline{f}}}}$ & $\mathrm{C}_{\mathrm{v}}=\alpha \sqrt{\frac{\mathrm{k}_{\mathrm{v}} \mathrm{E}}{\mathrm{f}_{\mathrm{yw}}}} / \frac{\mathrm{h}_{\mathrm{w}}}{\mathrm{t}_{\mathrm{w}}}$ & \\
\hline$\frac{h_{w}}{t_{w}}>\beta \sqrt{\frac{k_{v} E}{f_{y w}}}$ & $\mathrm{C}_{\mathrm{v}}-\frac{\gamma E \mathrm{k}_{\mathrm{v}}}{\left(\frac{\mathrm{h}_{\mathrm{w}}}{\mathrm{t}_{\mathrm{w}}}\right)^{2} \mathrm{f}_{\mathrm{yw}}}$ & \\
\hline
\end{tabular}

Tabla 1. Coeficiente de abolladura $\mathrm{Cv}$.

Para los casos con dicha relación de aspecto, se utiliza un coeficiente de abolladura $\mathrm{k}_{\mathrm{v}}=5, \mathrm{o}$.

\subsection{AASHTO}

La resistencia a abolladura por cortante de paneles de vigas armadas se define como $\mathrm{V}_{\mathrm{t}}$. Para paneles extremos no se considera la resistencia post-crítica [6] mientras que para paneles intermedios sí se considera [7]. No se limita explícitamente la utilización del campo de tracción para vigas híbridas. Asimismo, el coeficiente $\mathrm{C}_{\mathrm{v}}$ se obtiene a partir de la Tabla 1.

$$
V_{t}=0,58 t_{w} h_{w} f_{y w} C_{v}
$$

$$
V_{t}=0,58 \cdot t_{w} h_{w} f_{y w}\left[C_{v}+\frac{0,87\left(1-C_{v}\right)}{\sqrt{1+\left(\frac{a}{h_{w}}\right)^{2}}}\right]
$$

3.3. AISC

La resistencia a abolladura por cortante de paneles de vigas armadas se define como $V_{t}$ y puede o no incluir la resistencia post-crítica [8] [9], respectivamente. El coeficiente $\mathrm{C}_{\mathrm{v}}$ se incluye en la Tabla 1 . No se permite la utilización de la reserva post-crítica en:

- Paneles extremos en todos los elementos con rigidización transversal.

- Elementos cuando a $/ \mathrm{h}_{\mathrm{w}}$ excede 3,0 o $\left[260 /\left(\mathrm{h} / \mathrm{t}_{\mathrm{w}}\right)\right]^{2}$. Para los casos con dicha relación de aspecto, se utiliza un coeficiente de abolladura $\mathrm{k}_{\mathrm{v}}=5,0$

- Cuando la relación $\left(2 \mathrm{~h}_{\mathrm{w}} \mathrm{t}_{\mathrm{w}}\right) /\left(\mathrm{t}_{\mathrm{f}} \cdot \mathrm{b}_{\mathrm{f}}\right)>2,5$

- Cuando la relación $h_{w} / b_{f}>6,0$

$$
V_{t}=0,6 t_{w} h_{w} f_{y w} C_{v}
$$
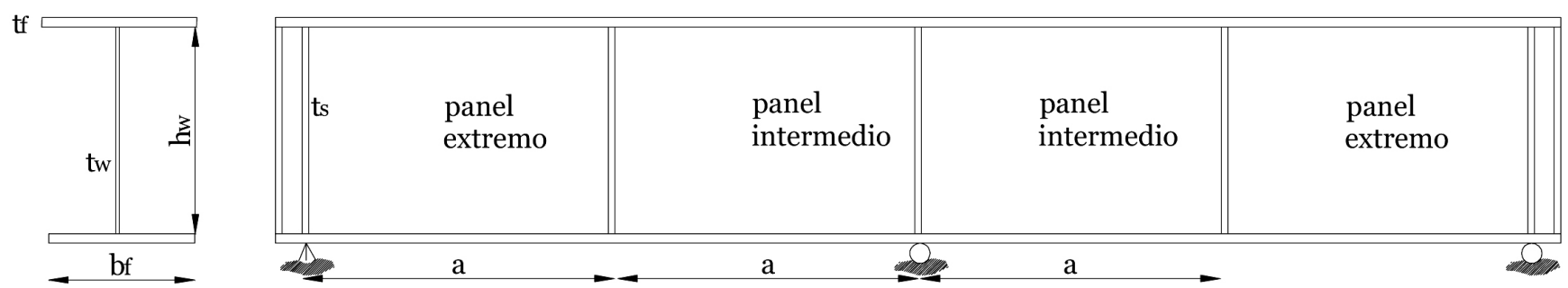

Figura 1. Variables geométricas en las piezas analizadas. 


$$
V_{t}=0,6 t_{w} h_{w} f_{y w}\left[C_{v}+\frac{1-C_{v}}{1,15 \sqrt{1+\left(\frac{a}{h_{w}}\right)^{2}}}\right]
$$

No se limita explícitamente la utilización de la resistencia post-crítica para vigas híbridas.

\subsection{EN1993-1-5 (23). EAE (24)}

La resistencia de cálculo frente a la abolladura por cortante se calcula mediante la contribución de dos términos, el alma $V_{b w, R d}$ y las alas $\mathrm{V}_{\mathrm{bffR}}$ [10], siendo $\gamma_{\mathrm{M} 1}$ el coeficiente parcial (se adopta un coeficiente parcial de seguridad $\gamma_{\mathrm{M} 1}=1$, 0 en este estudio).

$$
\mathrm{V}_{\mathrm{b}, \mathrm{Rd}}=\mathrm{V}_{\mathrm{bw}, \mathrm{Rd}}+\mathrm{V}_{\mathrm{bf}, \mathrm{Rd}} \leq \frac{1,2 \cdot \frac{\mathrm{f}_{\mathrm{yw}}}{\sqrt{3}} \mathrm{~h}_{\mathrm{w}} \mathrm{t}_{\mathrm{w}}}{\gamma_{\mathrm{M} 1}}
$$

La contribución del alma $\mathrm{V}_{\mathrm{bw}, \mathrm{Rd}}$ se define con [11] y la contribución de las alas se define con [12]. La Tabla 2 presenta las condiciones establecidas para obtener el coeficiente $\chi_{w}$.

$$
\begin{gathered}
\mathrm{V}_{\mathrm{bw}, \mathrm{Rd}}=\frac{\chi_{w} \frac{\mathrm{f}_{\mathrm{yw}}}{\sqrt{3}} \mathrm{~h}_{\mathrm{w}} \mathrm{t}_{\mathrm{w}}}{\gamma_{\mathrm{M} 1}} \\
\mathrm{~V}_{\mathrm{bf}, \mathrm{Rd}}=\frac{\mathrm{b}_{\mathrm{f}} \mathrm{t}_{\mathrm{f}}^{2} \mathrm{f}_{\mathrm{yf}}}{\mathrm{c} \gamma_{\mathrm{M} 1}}\left[1-\left(\frac{\mathrm{M}_{\mathrm{ed}}}{\mathrm{M}_{\mathrm{f}, \mathrm{Rd}}}\right)^{2}\right]
\end{gathered}
$$

Donde $\mathrm{b}_{\mathrm{f}} \mathrm{yt}_{\mathrm{f}}$ se toman del ala que conduce a la resistencia más baja, siendo $b_{f}$ no mayor que $15 \cdot\left(235 / f_{y w}\right)^{0,5}$. $t_{f}$ a cada lado del alma, $\mathrm{M}_{\mathrm{f}, \mathrm{Rd}}=\mathrm{M}_{\mathrm{f}, \mathrm{Rk}} / \gamma_{\mathrm{M} 1}$, es la resistencia de cálculo a flexión de la sección transversal considerado exclusivamente la sección reducida de las alas y «c» [13] es la distancia de anclaje del campo diagonal de tracciones en el ala.

$$
c=a\left(0,25+\frac{1.6 \cdot b_{f} \cdot t_{f}{ }^{2} \cdot f_{y f}}{t_{w} \cdot h_{w}{ }^{2} \cdot f_{y w}}\right)
$$

\subsection{Comparativa y análisis de las normativas}

Las normativas COVENIN, AISC y AASHTO se basan en la formulación de Basler para tener en cuenta la resistencia postcrítica de las vigas. Observando las mismas con detalle, se pun- tualiza que los coeficientes $\alpha, \beta$ y $\gamma$ son ligeramente distintos para una u otra. Dichos coeficientes se indican en la Tabla 3.

Es importante resaltar que en AISC y AASHTO, no se prohíbe la utilización de la resistencia post-crítica para vigas híbridas, mientras que COVENIN 1618-98 sí lo prohíbe explícitamente. Sin embargo, según las normativas AISC, AASHTO, una viga armada híbrida con una relación entre límites elásticos $f_{y f}>f_{y w}$ tiene como mucho la misma resistencia a cortante que su equivalente homogénea en la que $f_{y f}=f_{y w}$.

Por otra parte, al observar la diferencia fundamental entre las formulaciones Americanas y Europeas, se puntualizan varios aspectos:

- Las normativas Europeas se basan en el modelo del campo girado de tensiones de Höglund para obtener la resistencia a cortante en una viga armada a diferencia de las Americanas que se basan en el modelo de Basler de resistencia post-crítica.

- La formulación Europea permite incluir de manera explícita la contribución de las alas en la resistencia a cortante. De esta manera, una viga armada híbrida con una relación entre límites elásticos $\mathrm{f}_{\mathrm{yf}}>\mathrm{f}_{\mathrm{yw}}$ puede tener mayor resistencia a cortante que su equivalente homogénea en la que $\mathrm{f}_{\mathrm{yf}}=\mathrm{f}_{\mathrm{yw}}$.

- La formulación Europea concede la capacidad de anclaje del campo diagonal a paneles extremos si se proporciona la rigidez necesaria a los mismos. Las normativas americanas no permiten el desarrollo de la capacidad post-crítica en paneles extremos independientemente de su capacidad de anclaje.

- En el cálculo del coeficiente de abolladura $\mathrm{k}_{\mathrm{v}}$, en todas las normativas se considera el caso conservador de paneles simplemente apoyados.

\section{MODELIZACIÓN NUMÉRICA}

\subsection{Aspectos generales}

Se utiliza un modelo tridimensional de elementos finitos tipo Shell (25) contrastado con la experimentación para diferen-

Tabla 3. Coeficientes de las formulaciones de diferentes normativas.

\begin{tabular}{|l|c|c|c|}
\hline Normativa & $\boldsymbol{\alpha}$ & $\boldsymbol{\beta}$ & $\boldsymbol{\gamma}$ \\
\hline COVENIN-MINDUR 1618-98 & 1,08 & 1,35 & 1,48 \\
\hline AISC 2005 & 1,10 & 1,37 & 1,51 \\
\hline AASHTO-LRFD-2005 & 1,12 & 1,4 & 1,57 \\
\hline
\end{tabular}

Tabla 2. Coeficiente de reducción de la capacidad plástica del alma $\chi_{w}$.

\begin{tabular}{|c|c|c|c|}
\hline Condición de $\lambda_{\mathrm{w}}$ & $\begin{array}{c}\mathrm{\chi}_{\mathrm{w}} \text { panel de } \\
\text { extremo rígido }\end{array}$ & $\begin{array}{c}\mathrm{\chi}_{\mathrm{w}} \text { panel de } \\
\text { extremo no rígido }\end{array}$ & $\mathbf{k}_{\mathrm{v}}$ \\
\hline$\overline{\lambda_{\mathrm{w}}}<\frac{\mathrm{o}, 83}{\eta}$ & $\eta$ & $\eta$ & $\mathrm{k}_{\mathrm{v}}=5,34+4,0\left(\frac{\mathrm{h}_{\mathrm{w}}}{\mathrm{a}}\right)^{2}$ para $\frac{\mathrm{a}}{\mathrm{h}_{\mathrm{v}}} \geq 1,0$ \\
$\frac{0,83}{\eta} \leq \overline{\lambda_{\mathrm{w}}}<1,08$ & $\frac{0,83}{\overline{\lambda_{\mathrm{w}}}}$ & $\frac{\mathrm{o}, 83}{\overline{\lambda_{\mathrm{w}}}}$ & $\mathrm{k}_{\mathrm{v}}=4,0+5,34\left(\frac{\mathrm{h}_{\mathrm{w}}}{\mathrm{a}}\right)^{2}$ para $\frac{\mathrm{a}}{\mathrm{h}_{\mathrm{v}}}<1,0$ \\
\cline { 3 - 4 } & & $\frac{0,83}{\overline{\lambda_{\mathrm{w}}}}$ & $\overline{\lambda_{\mathrm{w}}}=\frac{\mathrm{h}_{\mathrm{w}}}{34,7 \mathrm{t}_{\mathrm{w}} \varepsilon \sqrt{\mathrm{k}_{\mathrm{v}}}}$ \\
\hline
\end{tabular}


tes fenómenos asociados a la abolladura de chapas (26) (27). El código permite reproducir la no linealidad geométricas y del material. La modelización se realiza siguiendo lo establecido en el Anejo C del Eurocódigo 3-1-5 (23) el cual sugiere recomendaciones para el uso del Método de los Elementos Finitos en el cálculo de estructuras de chapa de acero.

\subsection{Geometría}

Se plantea una geometría tridimensional para las diferentes chapas metálicas que conforman las vigas armadas utilizando elementos cuadriláteros tipo shell $\mathrm{S} 4 \mathrm{R}$ de primer orden con integración reducida. Se trata de elementos multipropósito con 6 grados de libertad por nodo.

\subsection{Material}

La ecuación constitutiva adoptada para el acero es elastoplástica perfecta y elasto-plástica con endurecimiento por deformación del tipo isotrópico. El módulo de elasticidad del acero utilizado es $\mathrm{E}=210000 \mathrm{~N} / \mathrm{mm}^{2}$ con un valor del coeficiente de Poisson $v=0,30$. Para estados multi-axiales, se introduce una superficie de fallo de von Mises.

\subsection{Introducción de carga y condiciones de contorno}

Se plantean vigas simplemente apoyadas con rigidización transversal en cada uno de los apoyos y en la zona de aplicación de carga. La viga consta de dos paneles de relación canto/ancho tal que las piezas no experimentan esfuerzos de flexión considerables. Los apoyos se simulan a lo largo de las alas inferiores en la cual se impiden los desplazamientos en los grados de libertad correspondientes. La carga se introduce como una presión uniforme en un área concentrada del ala superior.

\subsection{Tipo de análisis}

El estudio que se presenta requiere la utilización de estrategias de obtención de autovalores así como análisis no lineales a partir de los cuales se pueda inferir una curva de respuesta realista de una pieza sometida a solicitaciones de cortante. Ambas herramientas son fundamentales para estudiar el mecanismo de fallo de una viga.

\subsubsection{Análisis de autovalores}

Este tipo de análisis permite conocer las cargas críticas de inestabilidad de los sistemas elásticos así como la deformada afín a cada uno de ellos.

\subsubsection{Análisis no lineal}

El análisis no lineal realizado en las simulaciones incluye la no linealidad del material así como la no linealidad de la geometría. Se destaca que el proceso iterativo utilizado para la resolución del sistema es Newton-Raphson, mientras que la técnica incremental escogida es la longitud de arco (arclength).

\subsection{Imperfecciones}

Para poder reproducir adecuadamente la curva de respuesta no lineal, el modelo que se plantea debe incluir una serie de imperfecciones estructurales y geométricas. EN1993-1-5 en su anejo $\mathrm{C}$ recomienda utilizar imperfecciones geométricas afines a los modos de pandeo para el análisis numérico de vigas armadas (así como otras estructuras conformadas por chapas de acero). El procedimiento que establece EN1993-15 es el siguiente:

- Realizar un análisis de autovalores en una pieza ideal sometida a solicitaciones de cortante. Con este análisis se obtienen «n» modos de pandeo.

- Generar un modelo nuevo para el análisis no lineal cuya geometría se obtiene como una combinación lineal de los diferentes modos de pandeo establecidos previamente.

- EN1993-1-5. Anejo C sugiere escalar los modos obtenidos con una magnitud asociada a la tolerancia de fabricación de las piezas. La tolerancia de fabricación de vigas armadas difiere según las diferentes normativas. En este trabajo se utiliza como magnitud de escala el mínimo valor existente entre:

- $80 \%$ del espesor de alma $t_{w}$

- 80\% de la relación $h_{w} / 100$.

- Adicionalmente, según las recomendaciones de EN1993-15, se debería incluir algún patrón de tensiones residuales asociadas a la soldadura de las piezas. Sin embargo, dichas recomendaciones no sugieren ni perfiles ni valores concretos de tensión residual a ser utilizados. En el presente trabajo no se incluyen dichas imperfecciones. Se destaca la necesidad de investigar sobre el tema.

\subsection{Validación}

El modelo se validó utilizando dos especímenes publicados en estudios previos (28). La validación incluye un estudio de sensibilidad de malla, un estudio de cargas últimas y un estudio de la curva de respuesta. Las características específicas así como las condiciones realizadas para la validación se describen en (16).

\section{BASES DE DATOS}

\subsection{Base de datos experimental}

Se cuenta con un total de 53 vigas ensayadas en distintos laboratorios a nivel mundial (de tipología híbrida, entendiéndose que la relación $\left.f_{y f} / f_{y w}>1,20\right)$. La muestra representa la totalidad de ensayos localizables de los que se dispone información adecuada para el estudio (16) (22).

\subsection{Base de datos numérica}

La base de datos numérica se construye atendiendo a una variación paramétrica amplia inspirada en las dimensiones habituales de puentes metálicos y mixtos Europeos (15). Se plantea una variación utilizando tres relaciones $\mathrm{a} / \mathrm{h}_{\mathrm{w}}$, cinco esbelteces $h_{w} / t_{w}$ y cinco relaciones de hibridez $f_{y f} / f_{y w}$. Se cuenta entonces con una totalidad de 75 modelos de vigas híbridas sometidas a solicitaciones de cortante. En la Tabla 4 se presentan las características de las vigas estudiadas. Se diferencian tres grupos (I, II, y III) de 25 vigas cada uno. Los mismos se dividen en dos subgrupos A y B. Esta última división se realiza para analizar separadamente los especímenes confeccionados con aceros convencionales (S275, S355 y S460) y los aceros de alto lími- 
Tabla 4. Magnitudes adoptadas para los 75 prototipos de la base de datos numérica.

\begin{tabular}{|c|c|c|c|c|c|c|}
\hline \multirow{3}{*}{ Parámetros } & \multicolumn{6}{|c|}{ Grupos } \\
\hline & \multicolumn{2}{|c|}{ I } & \multicolumn{2}{|c|}{ II } & \multicolumn{2}{|c|}{ III } \\
\hline & $\mathbf{A}$ & B & $\mathbf{A}$ & B & $\mathbf{A}$ & $\mathbf{B}$ \\
\hline $\mathrm{f}_{\mathrm{yf}}\left(\mathrm{N} / \mathrm{mm}^{2}\right)$ & 275 & 460 & 275 & 460 & 275 & 460 \\
\hline \multirow{3}{*}{$\mathrm{f}_{\mathrm{yw}}\left(\mathrm{N} / \mathrm{mm}^{2}\right)$} & 275 & 460 & 275 & 460 & 275 & 460 \\
\hline & 355 & \multirow{2}{*}{690} & 355 & \multirow{2}{*}{690} & 355 & \multirow{2}{*}{690} \\
\hline & 460 & & 460 & & 460 & \\
\hline $\mathrm{h}_{\mathrm{w}}(\mathrm{mm})$ & \multicolumn{2}{|c|}{1000} & \multicolumn{2}{|c|}{1000} & \multicolumn{2}{|c|}{1000} \\
\hline $\mathrm{a}(\mathrm{mm})$ & \multicolumn{2}{|c|}{1000} & \multicolumn{2}{|c|}{2000} & \multicolumn{2}{|c|}{3000} \\
\hline $\mathrm{a} / \mathrm{h}_{\mathrm{w}}$ & \multicolumn{2}{|c|}{1,0} & \multicolumn{2}{|c|}{2,0} & \multicolumn{2}{|c|}{3,0} \\
\hline \multirow{5}{*}{$\mathrm{t}_{\mathrm{w}}(\mathrm{mm})$} & \multicolumn{2}{|c|}{6} & \multicolumn{2}{|c|}{6} & \multicolumn{2}{|c|}{6} \\
\hline & \multicolumn{2}{|c|}{8} & \multicolumn{2}{|c|}{8} & \multicolumn{2}{|c|}{8} \\
\hline & \multicolumn{2}{|c|}{10} & \multicolumn{2}{|c|}{10} & \multicolumn{2}{|c|}{10} \\
\hline & \multicolumn{2}{|c|}{12} & \multicolumn{2}{|c|}{12} & \multicolumn{2}{|c|}{12} \\
\hline & \multicolumn{2}{|c|}{15} & \multicolumn{2}{|c|}{15} & \multicolumn{2}{|c|}{15} \\
\hline & \multicolumn{2}{|c|}{30} & & & & \\
\hline & & & & & & \\
\hline$t_{f}=t_{s}(m m)$ & & & & & & \\
\hline & & & & & & \\
\hline & & & & & & \\
\hline Total por grupo & & & & & & \\
\hline
\end{tabular}

te elástico (S460 y S690). En cada subgrupo se utiliza como patrón de comparación una viga homogénea por cada $\mathrm{f}_{\mathrm{yw}}$ existente. Cabe destacar que el estudio se realiza con aceros que se expresan en nomenclatura europea. Para obtener una rápida equivalencia con las unidades utilizadas en Venezuela actualmente basta utilizar la relación $1 \mathrm{~N} / \mathrm{mm}^{2} \approx 10 \mathrm{Kg} / \mathrm{cm}^{2}$.

\section{RESULTADOS}

\subsection{Resultados experimentales}

La Figura 2 (a) muestra los resultados obtenidos experimentalmente de cortante último $\mathrm{V}_{\exp }$ normalizados a la resistencia plástica a cortante del alma $\mathrm{V}_{\text {plástica }}=\mathrm{t}_{\mathrm{w}} \cdot \mathrm{h}_{\mathrm{w}} \cdot \mathrm{f}_{\mathrm{yw}} \cdot(3)^{0,5}$. La amplia mayoría de los prototipos tiene un cortante último inferior a la resistencia plástica del alma (son almas bastante esbeltas y en su mayoría abollan). Por otra parte, la Figura 2 (b) muestra los resultados obtenidos experimentalmente de cortante último $\mathrm{V}_{\text {exp }}$ pero en este caso normalizados al cortante crítico $\mathrm{V}_{\mathrm{cri}}=\mathrm{t}_{\mathrm{w}} \cdot \mathrm{h}_{\mathrm{w}}$ $\cdot \tau_{\text {cri }}$ En este caso, los valores obtenidos reflejan en gran medida que la capacidad real de las piezas excede sustancialmente la carga crítica de abolladura. La carga máxima real de las piezas se encuentra en la gran mayoría de los casos entre la capacidad netamente plástica, y la carga asociada a la inestabilidad (carga crítica). Por otra parte, al no disponer de variación paramétrica puntual en la que vigas idénticas presenten distintos límites elásticos en las alas, los resultados experimentales no permiten extraer conclusiones asociadas a la influencia de la hibridez en la capacidad última a cortante. Sin embargo si permiten ilustrar que la capacidad última de las piezas está muy por encima de la carga crítica de abolladura incluso en vigas híbridas.

\subsection{Resultados numéricos}

La base de datos numérica ofrece versatilidad para el análisis de resultados. De la misma se pueden extraer curvas de respuesta de cada prototipo, carga última, evolución tensional, etc. La Figura 3 muestra la curva de respuesta de un espécimen cuya relación de aspecto $\mathrm{a} / \mathrm{h}_{\mathrm{w}}=1,0$. La Figura 4 muestra
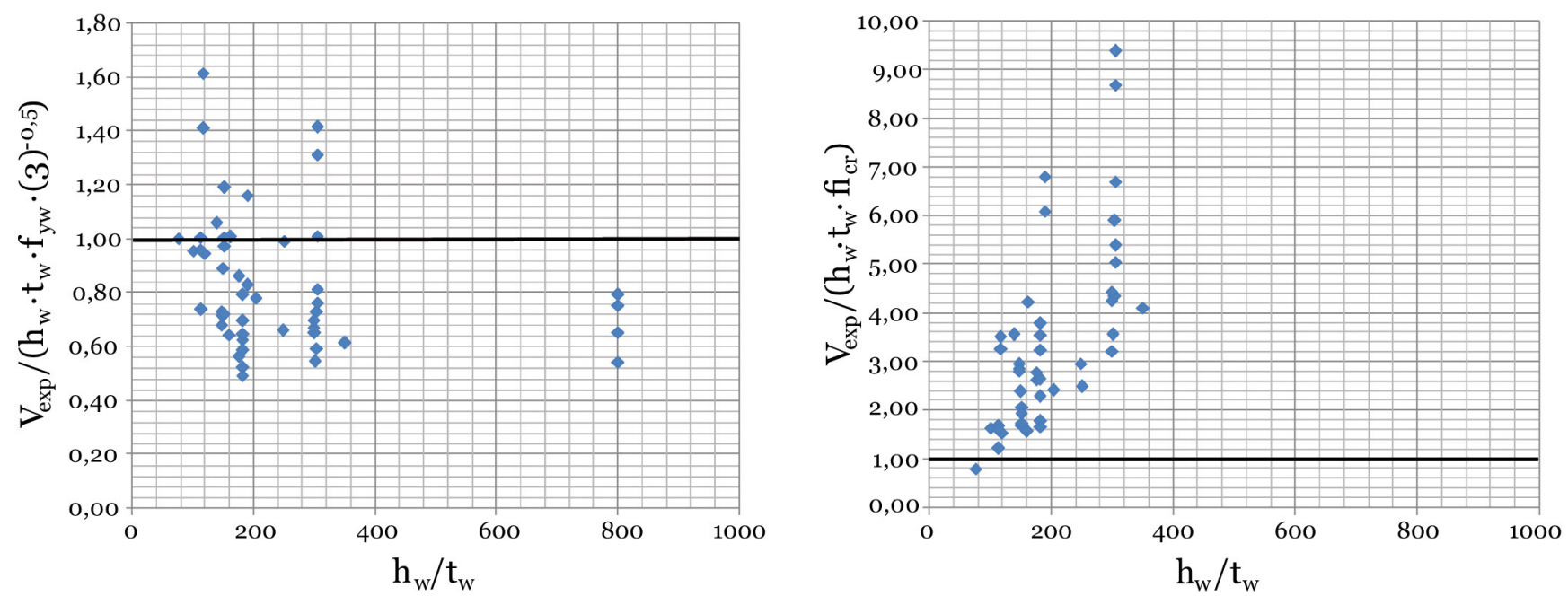

Figura 2. (a) $V_{\text {exp }} / V_{\text {plástico }}$ vs esbeltez del panel (b) $V_{\text {exp }} / V_{\text {crítico }}$ vs esbeltez del panel. 


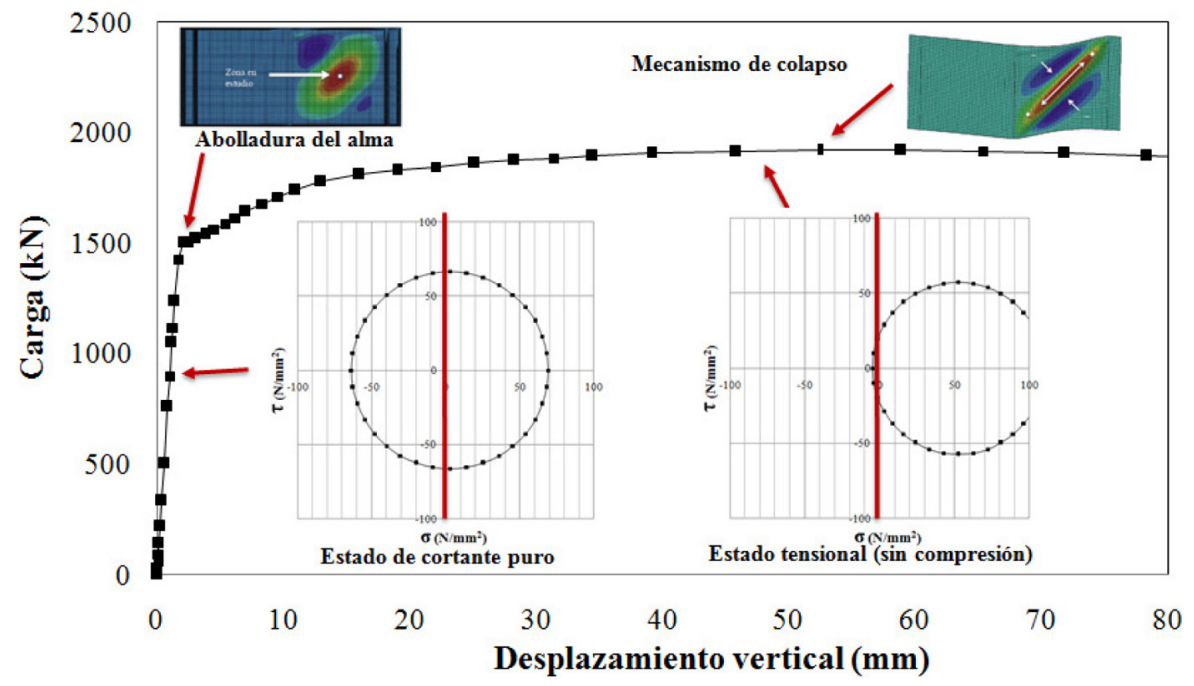

Figura 3. Curva de respuesta de prototipo con $\mathrm{a} / \mathrm{h}_{\mathrm{w}}=1,0 \mathrm{y} \mathrm{h}_{\mathrm{w}} / \mathrm{t}_{\mathrm{w}}=166,67$ y círculo de Mohr para dos incrementos de carga.

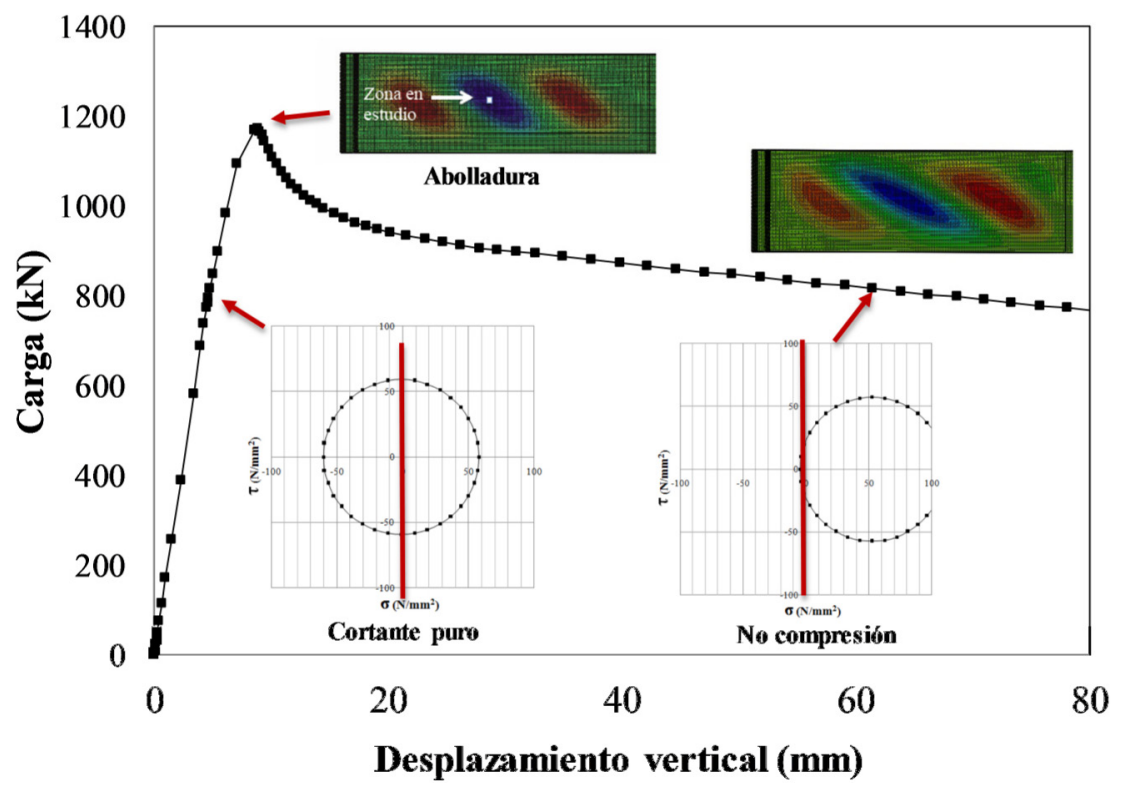

Figura 4. Curva de respuesta de prototipo con $\mathrm{a} / \mathrm{h}_{\mathrm{w}}=3,0 \mathrm{y} \mathrm{h}_{\mathrm{w}} / \mathrm{t}_{\mathrm{w}}=166,67$ y círculo de Mohr para dos incrementos de carga.

una curva de respuesta similar pero en este caso de un prototipo cuya relación de aspecto es $\mathrm{a} / \mathrm{h}_{\mathrm{w}}=3,0$.

Para el primer caso, se observa una rama lineal, en la cual el estado tensional de un elemento en el centro del panel es de cortante puro (nótese el círculo de Mohr asociado a esta rama extraído de un elemento finito en el centro del panel). En el momento en el que se pierde la linealidad de la curva de respuesta, la pieza abolla con una onda y el mecanismo resistente cambia. La pieza experimenta un incremento de carga hasta un máximo. Nótese que en este último tramo el círculo de Mohr asociado no indica un estado de cortante puro sino que se observa un estado tensional sin componentes de compresión. En estado de colapso, el mecanismo de fallo que se muestra indica la formación de un marco en el panel abollado que se ancla en las alas y rigidizadores, tal como se explica en los modelos teóricos descritos en el apartado 2.

Para el segundo caso $\left(a / h_{w}=3,0\right)$, se observa una rama lineal, en la cual el estado tensional de un elemento en el cen- tro del panel es de cortante puro. En este caso la pieza abolla con 3 ondas. La pendiente de la curva carga-desplazamiento cambia ligeramente a partir de esta abolladura y la pieza experimenta cierto incremento de carga pero luego cae abruptamente. El círculo de Mohr asociado indica que no existen componentes de compresión.

Se observa igualmente que los resultados obtenidos con los prototipos de $\mathrm{a} / \mathrm{h}_{\mathrm{w}}=2,0$ en algunos casos se acercan más a los de $\mathrm{a} / \mathrm{h}_{\mathrm{w}}=1,0$ mientras que en otros $\mathrm{a} \mathrm{a} / \mathrm{h}_{\mathrm{w}}=3,0$ (según sea su esbeltez del panel de alma).

\subsection{Influencia de la hibridez $f_{y f} / f_{y w}$ en la capacidad última}

A continuación se presentan distintas curvas de respuesta de vigas homogéneas e híbridas confeccionadas con idénticas características geométricas $\mathrm{a} / \mathrm{h}_{\mathrm{w}} \mathrm{y} \mathrm{h}_{\mathrm{w}} / \mathrm{t}_{\mathrm{w}}$ pero con distintas configuraciones de material. La Figura 5 muestra las curvas de respuesta de distintos prototipos híbridos y homogéneos 
de esbeltez considerable $\left(\mathrm{h}_{\mathrm{w}} / \mathrm{t}_{\mathrm{w}}=166,66\right)$ y de relación de aspecto $\mathrm{a} / \mathrm{h}_{\mathrm{w}}=1,0$. Los prototipos muestran de manera consistente las siguientes características:

- Una rama lineal.

- Una pérdida de linealidad asociada a la abolladura y cambio de mecanismo resistente que supone un anclaje del campo diagonal en alas y rigidizadores.

- Para límites elásticos de ala distintos, se observa una carga última distinta, lo que permite concluir que la capacidad resistente de las alas influye en la capacidad a cortante de las vigas.

- Las vigas de alto límite elástico (S690 y S460) tienen una capacidad post-crítica creciente debido a que en estos casos la ecuación constitutiva del material presenta endurecimiento por deformación.

La Figura 6 indica la curva de respuesta de prototipos de $\mathrm{a} / \mathrm{h}_{\mathrm{w}}=3, \mathrm{o}$ los cuales muestran de manera consistente las siguientes características:

- Una rama lineal.

- Una pérdida de linealidad asociada a la abolladura.

- Un valor de carga última que no es función del límite elástico de las alas en estudio (lo que indica la poca influencia de las mismas en el mecanismo resistente).

- Para límites elásticos de ala distintos, no se observa una carga última distinta, lo que permite concluir que la capacidad resistente de las alas no influye en la capacidad a cortante de las vigas.

- Una rama de reblandecimiento distinta según sea el límite elástico de las alas.

- Las vigas de alto límite elástico (S690 y S460) no tienen una capacidad post-crítica creciente a pesar de que en estos casos la ecuación constitutiva del material presenta endurecimiento por deformación.

La Figura 7 muestra la capacidad última de los 75 prototipos analizados normalizada a la carga última del prototipo homogéneo de idénticas características geométricas. Un valor superior a 1,0 indica que las vigas híbridas alcanzan valores superiores a las vigas homogéneas equivalentes. Se observa claramente que las vigas de relaciones de aspecto $\mathrm{a} / \mathrm{h}_{\mathrm{w}}=1,0 \mathrm{y}$ $\mathrm{a} / \mathrm{h}_{\mathrm{w}}=2,0$ presentan una capacidad a cortante que puede llegar a ser $20 \%$ superior en vigas híbridas. Para los casos de a/ $\mathrm{h}_{\mathrm{w}}=3,0$, aumentar el límite elástico de las alas no incrementa la capacidad última de las piezas a cortante.

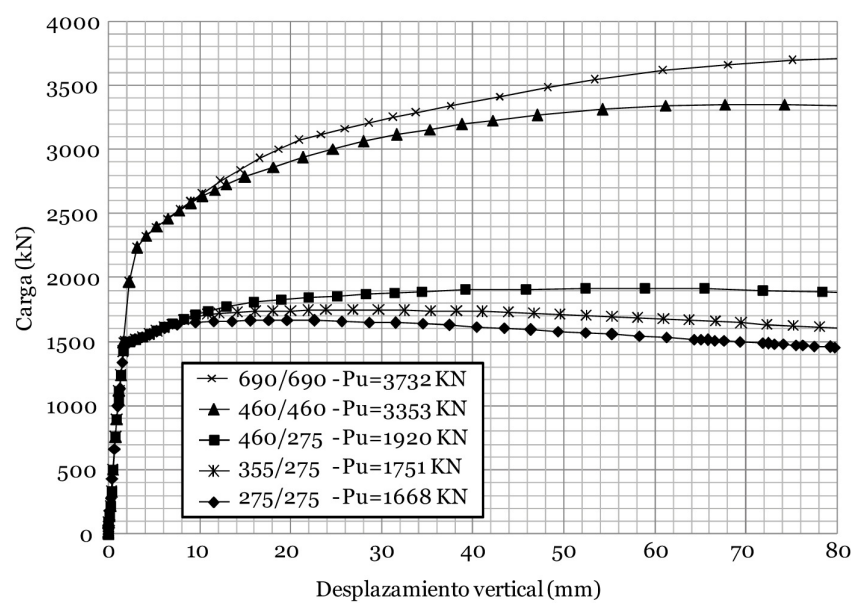

Figura 5. Curva de respuesta de prototipos híbridos con $\mathrm{h}_{\mathrm{w}} / \mathrm{t}_{\mathrm{w}}=166,66 \mathrm{y} \mathrm{a} / \mathrm{h}_{\mathrm{w}}=1,0$.

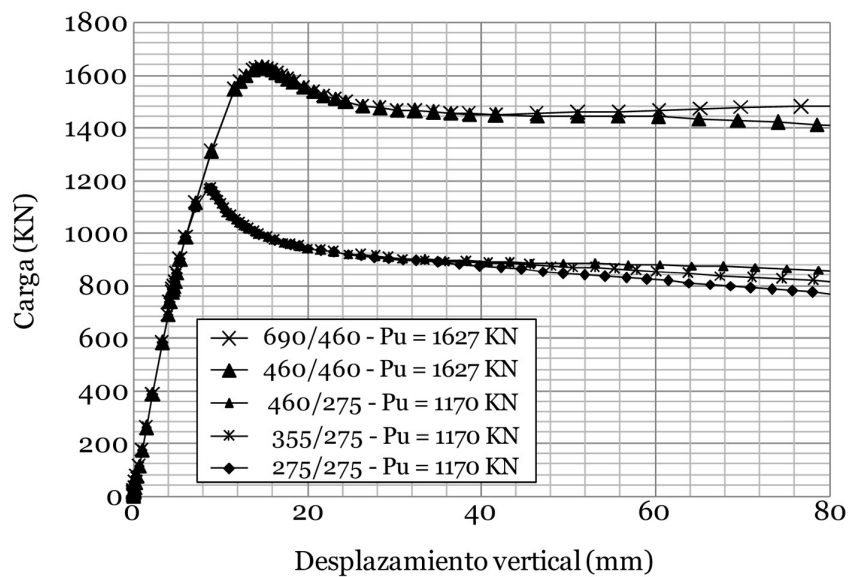

Figura 6. Curva de respuesta de prototipos híbridos con $\mathrm{h}_{\mathrm{w}} / \mathrm{t}_{\mathrm{w}}=166,66 \mathrm{y} \mathrm{a} / \mathrm{h}_{\mathrm{w}}=3,0$.

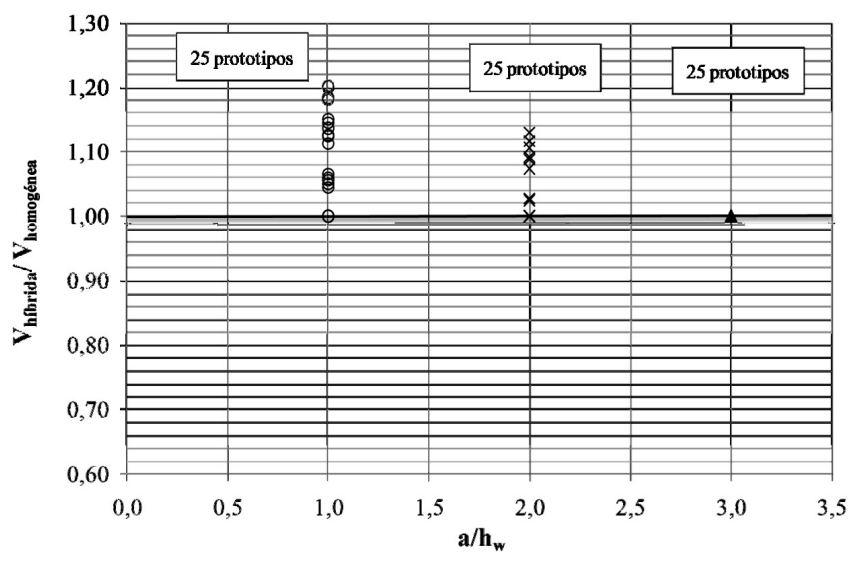

Figura 7. Cortante último de las vigas analizadas para distintos valores de $\mathrm{a} / \mathrm{h}_{\mathrm{w}}$.

\section{RESULTADOS NUMÉRICOS VS RESULTADOS NORMATIVOS}

Como base de comparación, se utiliza la relación $\mathrm{V}_{\text {numérico }} / \mathrm{V}_{\text {nor- }}$ mativo . Las Figuras 8 y 9 muestran la relación obtenida para las vigas de relación de aspecto $\mathrm{a} / \mathrm{h}_{\mathrm{w}}=1,0$ y 3,0 , respectivamente según las normativas estudiadas. Gráficamente, se puede observar que las relaciones obtenidas con EAE, AISC y AASHTO dependen consistentemente de la esbeltez del panel. Sin embargo, los valores obtenidos a partir de los resultados de COVENIN se alejan considerablemente de los numéricos.

Para las vigas de relación $\mathrm{a} / \mathrm{h}_{\mathrm{w}}=3,0$, la normativa AISC infravalora algunos resultados obtenidos en vigas muy esbeltas. Las formulaciones aplicables están limitadas a ciertos valores de esbeltez máxima según AISC lo que conlleva a limitar la aplicación del campo diagonal en vigas esbeltas.

Las Figuras 10 y 11 muestran los valores promedio obtenidos de la relación $\mathrm{V}_{\text {numérico }} / \mathrm{V}_{\text {normativo }}$ en función del parámetro $\mathrm{f}_{\mathrm{yf}} /$ $f_{\mathrm{yw}}$. Los resultados presentados se agrupan de manera separada en cada figura para las relaciones de aspecto $\mathrm{a} / \mathrm{h}_{\mathrm{w}}=1,0 \mathrm{y} \mathrm{a} /$ $h_{w}=3,0$ respectivamente.

Para las vigas con relación de aspecto $\mathrm{a} / \mathrm{h}_{\mathrm{w}}=1,0$, se observan las siguientes características:

- Los resultados promedio presentados se encuentran del lado de la seguridad. 


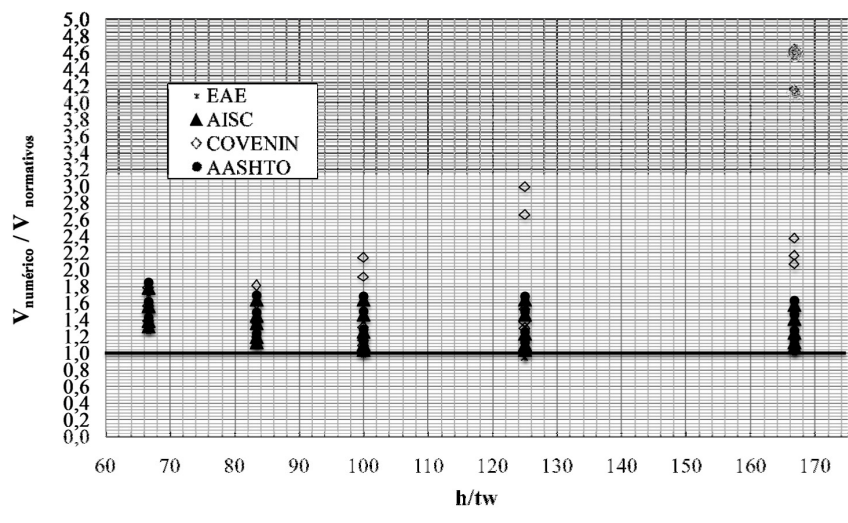

Figura 8. Cortante último de las vigas analizadas para distintas normativas. $\mathrm{a} / \mathrm{h}_{\mathrm{w}}=1,0$.

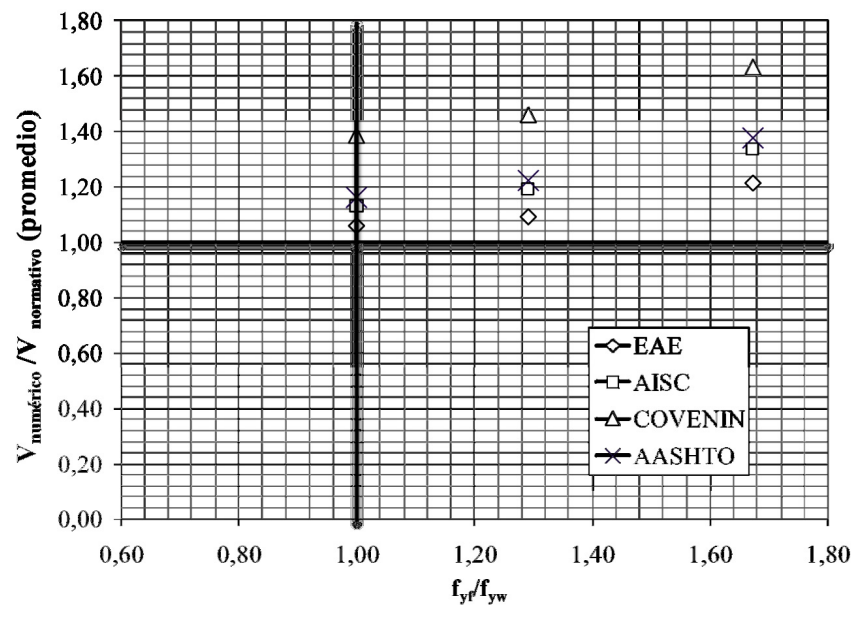

Figura 10. Relación $V_{\text {numérico }} / V_{\text {normativo }}$ en función de la hibridez $\mathrm{f}_{\mathrm{y} f} / \mathrm{f}_{\mathrm{yw}}$. $\mathrm{a} / \mathrm{h}_{\mathrm{w}}=1,0$.

- Conforme crece la hibridez, el modelo numérico arroja valores superiores a los derivados por las normativas.

- COVENIN arroja los resultados más conservadores mientras que la EAE arroja los resultados más ajustados.

Para las vigas con relación de aspecto $\mathrm{a} / \mathrm{h}_{\mathrm{w}}=3,0$, se observan las siguientes características:

- Las relaciones de cargas obtenidas con las normativas COVENIN, AISC y AASHTO no dependen de $f_{y f} / f_{y w}$ mientras que los resultados derivados de la EAE presentan una dependencia negativa.

- COVENIN arroja los resultados más conservadores mientras que la EAE arroja los resultados más ajustados.

\section{CONCLUSIONES}

El presente estudio describe el comportamiento estructural de las vigas armadas homogéneas e híbridas en sección doble T sometidas a solicitaciones de cortante. El estudio fenomenológico muestra el comportamiento post-crítico de las vigas de diferentes relaciones de aspecto de los paneles $a / h_{w}$. Se observa una sensibilidad considerable a dicho parámetro.

- En vigas de relación $\mathrm{a} / \mathrm{h}_{\mathrm{w}}=1,0$, se observa una reserva postcrítica asociada al campo diagonal de tracciones y a la formación de rótulas plásticas de las alas. En todos los casos estudiados, las vigas híbridas presentan una resistencia a cortante superior que las vigas homogéneas.

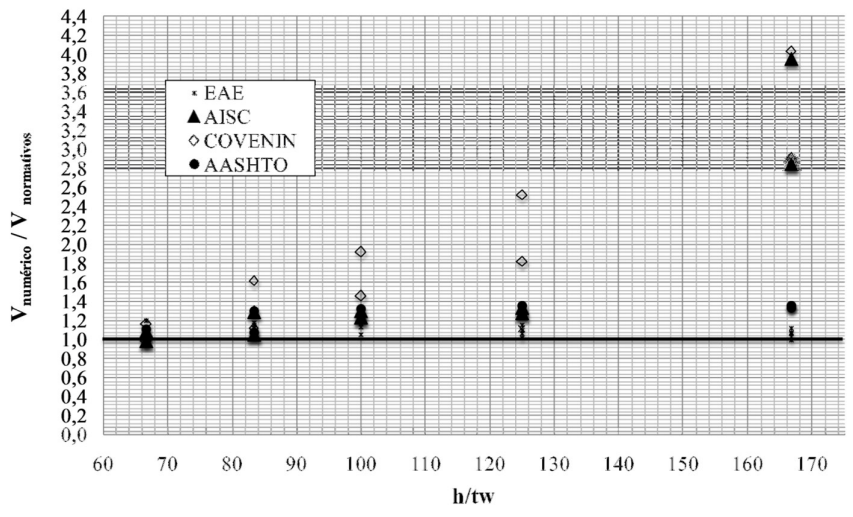

Figura 9. Cortante último de las vigas analizadas para distintas normativas. $\mathrm{a} / \mathrm{h}_{\mathrm{w}}=3,0$.

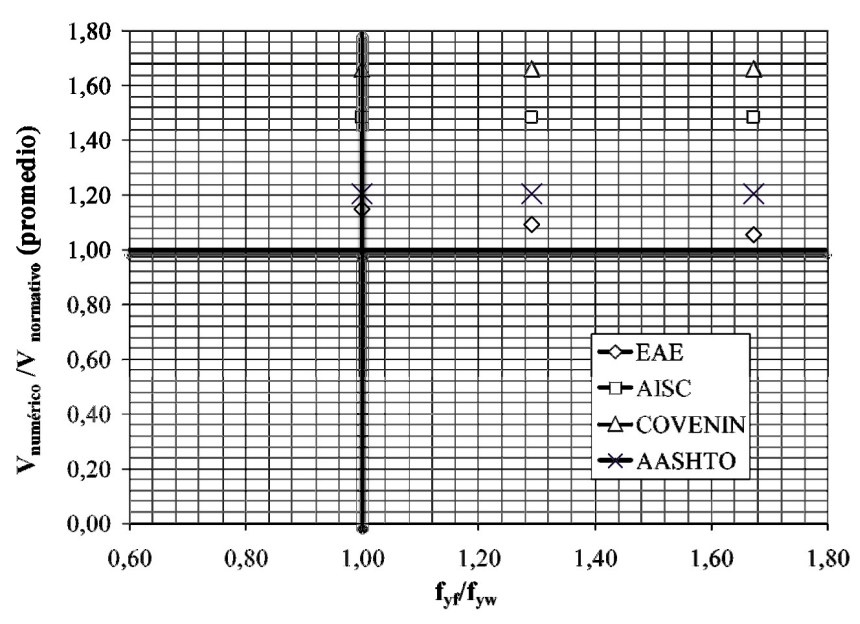

Figura 11. Relación $V_{\text {numérico }} / V_{\text {normativo }}$ en función de la hibridez $f_{y f} / f_{y w} \cdot a / h_{w}=3,0$.

- Para las vigas de relaciones $\mathrm{a} / \mathrm{h}_{\mathrm{w}}=3,0$ (y en la mayoría de los casos de $\mathrm{a} / \mathrm{h}_{\mathrm{w}}=2,0$ ) la capacidad post-crítica presenta una dependencia distinta con respecto a la formación de rótulas en las alas. En algunos casos estudiados, las vigas híbridas no presentan una resistencia a cortante superior que las vigas homogéneas.

Si se comparan los valores obtenidos con el modelo numérico con las normativas, se observan dos tendencias distintas:

- Para las vigas de relación $\mathrm{a} / \mathrm{h}_{\mathrm{w}}=1,0$, conforme crece la relación $\mathrm{f}_{\mathrm{yf}} / \mathrm{f}_{\mathrm{yw}}$, los valores que predicen las normativas se alejan gradualmente de los obtenidos con el modelo. En algunos casos, la diferencia llega a ser de 30\%. Los valores más conservadores son los que arroja COVENIN ya que la misma ni siquiera permite la utilización de la capacidad post-crítica que se puede desarrollar en las vigas sometidas a cortante.

- Para las vigas de relación $\mathrm{a} / \mathrm{h}_{\mathrm{w}}=2,0$ y a $/ \mathrm{h}_{\mathrm{w}}=3,0$, conforme crece la relación $\mathrm{f}_{\mathrm{yf}} / \mathrm{f}_{\mathrm{yw}}$, los valores que predicen las normativas AISC-AASHTO-COVENIN se mantienen de manera consistente. Sin embargo, EAE predice cierto crecimiento en la capacidad (ya que la misma si incluye de manera explícita el límite elástico de las alas) y en este caso no se obtienen resultados del todo seguros.

Los resultados obtenidos muestran discrepancias en los tratamientos normativos tanto a nivel cuantitativo como cualitativo. En todos los casos, se observa que la penalización que tienen las vigas híbridas según COVENIN en el cálculo de su resistencia a cortante es muy severa. Las vigas híbridas 
se comportan de manera similar a las homogéneas en todos los casos. Tanto los resultados experimentales como los resultados numéricos muestran que la capacidad última de las vigas armadas híbridas es sensiblemente superior a la carga crítica de abolladura. Se recomienda revisar la penalización que existe actualmente en el apartado 17.3 (a) de la normativa venezolana COVENIN-MINDUR para la utilización de la capacidad post-crítica en vigas híbridas en futuras actualizaciones. De esta manera se podría explotar de manera adecuada las ventajas estructurales y económicas que presenta esta tipología estructural. Finalmente, se sugiere profundizar en la influencia de $\mathrm{a} / \mathrm{h}$ en la capacidad última de vigas armadas sometidas a cortante.

\section{AGRADECIMIENTOS}

Los autores agradecen al Profesor Donald White del Georgia Institute of Technology por toda la documentación técnica proporcionada.

\section{REFERENCIAS}

(1) MinppTT-Instituto de Ferrocarriles del Estado. www.ife.gob.ve. Venezuela. Consultada el 12 de noviembre de 2013.

(2) Covenin. (1998). Covenin-Mindur 1618-98: Estructuras de acero para edificaciones. Método de los estados límite. Caracas: Comisión Venezolana de Normas Industriales.

(3) AISC. (2002). Load and resistance factor design specification for structural steel buildings (3rd Ed.). Chicago: American Institute of Steel Construction.

(4) AASHTO. (2005). LRFD Bridge Design Specification. Washington, D.C.: American Association of State Highway and Transportation Officials Inc.

(5) Veljkovic, M., Johansson, B. (2004). Design of hybrid steel girders. Journal of Constructional Steel Research, 64(3-5): 535-547, doi: http://dx.doi.org/10.1016/So143-974X(03)o0128-7.

(6) Barker, M.G., Schrage, S.D. (2000) High performance Steel Bridge Design and Cost Comparisons. Transportation Research Record, 1740: 33-39, doi: http://dx.doi.org/10.3141/1740-05.

(7) Frost, R., Schilling, C. (1964). Behaviour of hybrid beams subjected to static loads. Journal of the Structural Division, 90(3): 55-86.

(8) Nethercot, D. (1976). Buckling of welded hybrid steel I-beams. Journal of the structural division, 102(3): $461-474$.

(9) Greco, N., Earls, C. (2000). Structural Ductility in Hybrid High Performance Steel Beams. Journal of Structural Engineering, 129(12): 1584-1595, doi: http://dx.doi.org/10.1061/(ASCE)0733-9445(2003)129:12(1584).

(10) Chacón, R., Mirambell, E., Real, E. (2010). Hybrid steel plate girders subjected to patch loading, Part 1: Numerical study. Journal of Constructional Steel Research, 66(5): 695-708, doi: http://dx.doi.org/10.1016/j.jcsr.2009.12.005.

(11) Bitar, D. (2003). Résistance a la flexion des poutres hybrides à section en I. Construction Métallique, 40(2): 77-92.

(12) Carskaddan, P. (1968). Shear buckling of unstiffened hybrid beams. Journal of the Structural Division, 94(8): 1965-1990.

(13) Barker, M., Hurst, A., White, D. (2002). Tension Field Action in Hybrid Steel Girders. Engineering Journal, 39(1): 52-62.

(14) Azizinamini, A., Hash, J., Yakel, A., Farimani, R. (2007). Shear capacity of hybrid plate girders. Journal of Bridge Engineering, 12(5): 535-543, doi: http://dx.doi.org/10.1061/(ASCE)1084-0702(2007)12:5(535).

(15) ComBri. (2007).Competitive Steel and Composite Bridges by Improved Steel Plated Structures. Final Report, RFCS Contract No. RFS-CR-03018.

(16) Rojas, J. (2013).Vigas híbridas sometidas a solicitaciones de cortante. Propuesta de actualización de la Norma Venezolana COVENIN 1618:1998 (Tesis de Máster). Barcelona: Departamento de Ingeniería de la Construcción. ETSECCP, BarcelonaTech-UPC. http://upcommons.upc.edu/handle/2099.1/18448.

(17) Timoshenko, S.P. (1961). Theory of elastic stability (2nd edition). New York: McGraw-Hill Book Company.

(18) Basler, K. (1961). Strength of Plate Girders in Shear. Journal of Structural Division, 87(7): 151-180.

(19) Porter, D.M., Rockey, K.C., Evans, H.R. (1975). The Ultimate Load Behavior of Plate Girders Loaded in Shear. The Structural Engineer, 53(8): 313-325.

(20) Rockey, K.C., Skaloud, M. (1972). The Ultimate Load Behaviour of Plated Girders Loaded in Shear. The Structural Engineer, 50(1): 29-47.

(21) Höglund, T. (1971). Simply supported long thin plate I-girders without web stiffeners subjected to distributed transverse load. En IABSE Colloquium London, Reports of the Working Comissions, 11: 85-97.

(22) White, D. W., Barker, M. (2004). Shear strength of transversely-stiffened steel I-girders. Mechanics and Materials Report, No. 26. Atlanta: Structural Engineering, School of Civil and Environmental Engineering, Georgia Institute of Technology.

(23) CEN. (2005). EN 1993-1-5 Eurocode 3: design of steel structures - Part 1-5: Plated structural elements. European Committee for Standardization.

(24) EAE. (2012). Instrucción española de acero estructural. Madrid : Ministerio de Fomento.

(25) Abaqus. (2012). FEA v.6.10, Simmulia(C) DassaultSystèmes. http://www.3ds.com/products-services/simulia/products/.

(26) Chacon, R., Mirambell, E., Real, E. (2009). Influence of designer-assumed initial conditions of the numerical modelling of steel plate girders subjected to patch loading. Thin Walled Structures, 47(4): 391-402, doi: http://dx.doi. org/10.1016/j.tws.2008.09.001.

(27) Bedynek, A. ,Real, E. ,Mirambell, E. (2013). Tapered plate girders under shear: Tests and numerical research. Engineering Structures, 46(1): 350-358, doi: http://dx.doi.org/10.1016/j.engstruct.2012.07.023.

(28) Real, E., Chacón, R., Mirambell, E. (2008). Shear response of hybrid steel plate girders. En Proceedings of Eurosteel - $5^{\text {th }}$ European Conference of Steel and Composite Structures. Graz, Austria. 